T H E

\title{
POLITICAL SONGS
}

\author{
OF ENGLAND.
}


T H E

\section{P OL I T C A L S O N G \\ OF E N G LA ND,}

FROM THE REIGN OF JOHN TO THAT OF EDWARD II.

EDTED AND TRANSLATED

BY THOMAS WRIGHT, Esq., M.A., F.S.A., \&c. OF TRINITY COLEEGE, CAMBRIDGE.

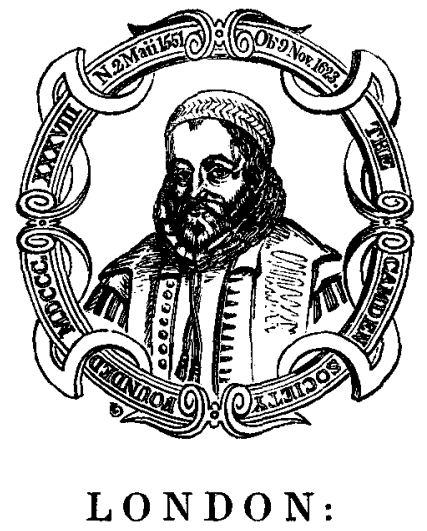

PRINTED FOR THE CAMDEN SOCIETY, BY JOHN BOWYER NICHOLS AND SON, PARLIAMENT STREET. M. $\overline{D C C C . X X X I X . ~}$ 


\section{COUNCIL}

OF

\section{THE CAMDEN SOCIETY,}

ELECTED MAY 2, 1839 .

\section{President,}

THE RIGHT HON. LORD FRANCIS EGERTON, M.P.

THOMAS AMYOT, ESQ. F.R.S. Treas. S.A. Director. THE REV. PHILIP BLISS, D.C.L., F.S.A., Registrar of the University of Oxford.

JOHN BRUCE, ESQ. F.S.A. Treasurer.

JOHN PAYNE COLLIER, ESQ. F.S.A.

C. PURTON COOPER, ESQ. Q.C., D.C.L., F.R.S., F.S.A. RT. HON. THOMAS PEREGRINE COURTENAY.

T. CROFTON CROKER, ESQ. F.S.A., M.R.I.A.

THE REV. ALEXANDER DYCE, B.A.

SIR HENRY ELLIS, K.H., F.R.S., Sec. S.A.

THE REV. JOSEPH HUNTER, F.S.A.

JOHN HERMAN MERIVALE, ESQ. F.S.A.

JOHN GAGE ROKEWODE, ESQ. F.R.S., Director S.A.

THOMAS STAPLETON, ESQ. F.S.A.

WILLIAM J. THOMS, ESQ. F.S.A. Secretary.

THOMAS WRIGHT, ESQ. M.A., F.S.A. 